\title{
Suppressor of Fused Is Required to Maintain the Multipotency of Neural Progenitor Cells in the Retina
}

\author{
Matt A. Cwinn, ${ }^{1,2}$ Chantal Mazerolle, ${ }^{1}$ Brian McNeill, ${ }^{1,2}$ Randy Ringuette, ${ }^{1,2}$ Sherry Thurig, ${ }^{1}$ Chi-chung Hui, ${ }^{3}$ \\ and Valerie A. Wallace ${ }^{1,2}$ \\ ${ }^{1}$ Vision Program, Ottawa Hospital Research Institute, Ottawa, Ontario K1H 8L6, Canada, ${ }^{2}$ Department of Ophthalmology and Department of Biochemistry, \\ Microbiology and Immunology, University of Ottawa, Ottawa, Ontario K1H 8M5, Canada, and ${ }^{3}$ Program in Developmental \& Stem Cell Biology, The \\ Hospital for Sick Children and Department of Molecular Genetics, University of Toronto, Toronto, Ontario M5G 1L7, Canada
}

The morphogen sonic hedgehog (Shh) plays a crucial role in development of the CNS, including the neural retina. Suppressor of fused (Sufu) has been recently identified as a critical regulator of Hh signaling in mammals. However, the precise roles that Sufu plays in the regulation of proliferation and cell-fate decisions in neural progenitors is unknown. Here, we have addressed these questions by conditionally deleting Sufu in mouse multipotent retinal progenitor cells (RPCs). Sufu deletion in RPCs results in transient increases in Hh activity and proliferation followed by developmentally premature cell-cycle exit. Importantly, we demonstrate a novel role for Sufu in the maintenance of multipotency in RPCs. Sufu-null RPCs downregulate transcription factors required to specify or maintain RPC identity $(R a x, V s x 2)$ and multipotency (Pax6) but continue to express the neural progenitor marker Sox2. These cells fail to express retinal lineage-specific transcription factors, such as Math5, and adopt an amacrine or horizontal cell fate at the expense of all other classes of retinal neurons. Genetic elimination of Gli2 in Sufu-null RPCs attenuates Hh pathway activity and restores multipotency in neural progenitors. These data provide novel evidence that $S u f u$-mediated antagonism of $\mathrm{Hh} / \mathrm{Gli} 2$ signaling is required to maintain RPC multipotency and identity.

\section{Introduction}

CNS development requires a complex interplay between cellextrinsic and -intrinsic signals that regulate stem cell/progenitor cell proliferation and diversification. The neural retina is an accessible and relevant model for interrogating the integration of these processes. The seven cell types in the adult retina develop in a temporal sequence from multipotent retinal progenitor cells (RPCs) (Young, 1985). RPC specification, proliferation, and neurogenic competence are regulated by several transcription factors, including Pax6 (Marquardt et al., 2001), Rax (Mathers et al., 1997), Vsx2 (Burmeister et al., 1996), Six6 (Li et al., 2002), and Sox2 (Taranova et al., 2006); retinal lineage commitment is regulated by the coordinated activity of additional transcription factors, including bHLH and homeobox genes (Ohsawa and Kageyama, 2008). Pax6 is the only gene identified to date that is required for RPC multipotency, as conditional Pax6 deletion at the optic cup stage causes RPCs to adopt an amacrine interneuron fate exclusively (Marquardt et al., 2001). While a number of

\footnotetext{
Received Oct. 8, 2010; revised Feb. 3, 2011; accepted Feb. 9, 2011.

Author contributions: M.A.C. and V.A.W. designed research; M.A.C., C.M., B.M., R.R., and S.T. performed research; M.A.C., C.M., and V.A.W. analyzed data; M.A.C. and V.A.W. wrote the paper; C.-C.H. contributed unpublished reagents/analytic tools.

This work was supported by an operating grant to V.A.W. from the Canadian Cancer Society (National Cancer Institute of Canada Grant 016435). Matt Cwinn is a recipient of a Natural Sciences and Engineering Council of Canada Masters Studentship.

Correspondence should be addressed to Valerie A. Wallace, Ottawa Hospital Research Institute, 501 Smyth Road, Ottawa, Ontario KIH 8L6, Canada. E-mail: vwallace@ohri.ca.

DOI:10.1523/JNEUROSCI.5495-10.2011

Copyright $\odot 2011$ the authors $\quad 0270-6474 / 11 / 315169-12 \$ 15.00 / 0$
}

cell-extrinsic cues, including Hedgehog (Hh) signaling, also play a role in RPC proliferation and cell-fate specification (Amato et al., 2004), the mechanism(s) that couple determination of retinal fate to extrinsic signaling is not known.

The secreted morphogen Sonic hedgehog (Shh) is a key regulator of CNS development. Binding of Shh to its receptor, Ptch, derepresses Smo and initiates the Hh signaling (Alcedo et al., 1996; Murone et al., 1999) that converges upon the Gli transcription factors (Gli1-3) and, ultimately, target gene transcription. Suppressor of fused (Sufu) is a key antagonist of Hh signaling in mammals (Cooper et al., 2005; Svard et al., 2006; Varjosalo et al., 2006) and exerts dichotomous effects on Gli function by both cytoplasmic sequestration and increased protein stability (Barnfield et al., 2005; Humke et al., 2010). Embryonic deletion of Sufu results in a strong Hh gain of function phenotype with extensive CNS mispatterning (Svard et al., 2006). However, the precise roles of Sufu in the regulation of Hh signaling in developing neuroepithelia are unknown.

In the developing retina, Shh is secreted by retinal ganglion cells (GCs) and signals to RPCs to regulate cell fate, proliferation, and self-renewal (Amato et al., 2004; Wang et al., 2005). Recently, Gli2 has been identified as an important mediator of Hh-induced RPC proliferation and cell fate (Wall et al., 2009). As Sufu likely integrates the cell-extrinsic Hh signal and cell-intrinsic Gli responses to direct progenitor behavior, we sought to determine the roles of Sufu in RPCs. Here, we demonstrate that Sufu negatively regulates Hh signaling and proliferation in RPCs. Unexpectedly, Sufu-null RPCs also failed to maintain their multipotency and differentiated exclusively as amacrine and horizontal neurons. This 
phenotype was characterized by the downregulation of transcription factors required to maintain RPC identity and multipotency, including Pax6, Rax, and Vsx2, and a failure to initiate expression of transcription factors required for the development of retinal cell lineages. We further demonstrate that these effects were mediated by Gli2. These findings identify a novel role for Sufu/Gli2 function upstream of Pax6 in the regulation of RPC multipotency.

\section{Materials and Methods}

Mice. All experiments were approved by the University of Ottawa Animal Care Ethics Committee and adhered to the guidelines of the Canadian Council on Animal Care. Both male and female mice were selected randomly for all genotypes. C57BL/6 mice were obtained from the Charles River Laboratory. The $\alpha \mathrm{PO}$-Cre transgenic mice, which express Crerecombinase under the control of the $\alpha \mathrm{P} 0$ element of the Pax 6 promoter, were obtained from Dr. P Gruss (Max-Planck Institute, Gottingen, Germany) (Marquardt et al., 2001) and were maintained on a C57BL/6 background. Sufu ${ }^{F / F}$ mice harbor loxP sites flanking Sufu exons 4-8 (Pospisilik et al., 2010) and were maintained on a C57BL/6 background. Retina-restricted conditional Sufu knock-out mice were generated by crossing $\alpha P 0$-Cre; $S u f u^{+/ F l}$ mice with $S u f u^{F / F}$ mice to generate Cre;Sufu ${ }^{F / F}$ mice. Gli2 mutant mice were maintained on a CD1 background and genotyped as published previously (Wall et al., 2009). ROSA Cre reporter mice were obtained from Jackson Laboratories. Mice were genotyped by the PCR of tail DNA for wild-type and floxed Sufu alleles with the following primers: forward (F)-5'CTGTTTGTACTCATGGTC3', reverse (R)-5' CCTACCCTTTCCAGTAAG3', Del-5' GCTGAATTCTTGACTCACTG3', Neo-5'GTGTCAGTTTCATCGCCTG3'. Primers directed against the wild-type allele yielded a band size of $300 \mathrm{bp}$, those directed against the floxed allele yielded a band size of 350bp.

Retinal explant culture, electroporation BrdU incorporation, and cell dissociation. Explants were prepared and electroporated as described previously (Matsuda and Cepko, 2004). Briefly, eyes from postnatal day 0 (P0) mice were dissected free of the optic nerve and RPE, and retinas were then electroporated (ECM 830 BTX Harvard apparatus) in a $2 \mathrm{~mm}$ gap cuvette (VWR). The DNA plasmids used in this study were Smo-M2 (CMV promoter) (a gift from G. Fishell, New York University, New York, NY), Sufu-Flag (CMV promoter) (Ding et al., 1999), pUB-GFP, Gli-Luciferase (Sasaki et al., 1997) (a gift from Dr. H Sasaki, Osaka, Japan), Renilla Luciferase (a gift from Dr. Alan Mears, Ottawa Hospital Research Institute, Ottawa, Canada), and Cre ( $\beta$-actin promoter). Plasmids were prepared using a Qiagen Maxiprep kit according to manufacturer's instructions (Qiagen) and were electroporated at a concentration of $\sim 1$ $\mu \mathrm{g} / \mu \mathrm{l}$. After electroporation, the lens was removed and explants were flattened on micropore filters (Millipore) and cultured in serum-free retinal explant medium (Wang et al., 2005). For BrdU incorporation experiments, explants were pulsed with BrdU (Sigma) for $5 \mathrm{~h}$ before enzymatic dissociation. To obtain single cell dissociates of retinal explants for IHC and fluorescent activated cell sorting (FACS) analysis, retinal explants were treated with $0.1 \mathrm{mg} / \mathrm{ml}$ trypsin (Sigma) in sterile calcium-free PBS (Invitrogen) at $37^{\circ} \mathrm{C}$ for $15 \mathrm{~min}$. Trypsinization was inhibited with $10 \%$ FBS/ DMEM/DNAseI ( $0.2 \mathrm{mg} / \mathrm{ml}$, Sigma) and tissue was triturated to obtain a single cell suspension. Cells were pelleted at $1000 \times g$ for $5 \mathrm{~min}$ and resuspended in $350 \mu \mathrm{l}$ of 10\%FBS/DMEM/ insulin $(0.01 \mathrm{mg} / \mathrm{ml}$, Sigma). Cell suspensions $(15 \mu \mathrm{l})$ were plated on glass slides, which were then incubated in a humidified chamber for 15 min at room temperature followed by $40 \mathrm{~min}$ at $37^{\circ} \mathrm{C}$ to allow the cells to adhere to the slides. Cells were then fixed with $4 \%$ PFA for 5 min, washed three times with sterile PBS and air dried. Cells were either processed immediately for immunocytochemistry or stored at $-20^{\circ} \mathrm{C}$.

Tissue preparation for in situ hybridization or immunohistochemistry. For timed matings, the morning of vaginal plug detection was considered embryonic day 0.5 (E0.5). To label S-phase cells in vivo, time-mated females were given two intraperitoneal injections $2 \mathrm{~h}$ apart of BrdU at 0.1 $\mathrm{mg} / \mathrm{g}$ body weight. For processing of embryonic retinae, whole heads were fixed in $4 \%$ PFA in PBS overnight at $4^{\circ} \mathrm{C}$. For fixation of adult retinae, mice were anesthetized using $\mathrm{CO}_{2}$, and a cardiac perfusion was performed using $\sim 10 \mathrm{ml}$ of $4 \%$ PFA. Eyes were then enucleated and fixed in $4 \%$ PFA overnight. Tissue was then washed $3 \times 10 \mathrm{~min}$ in sterile PBS $\left(0.14 \mathrm{M} \mathrm{NaCl}, 2.5 \mathrm{~mm} \mathrm{KCl}, 0.2 \mathrm{M} \mathrm{Na}_{2} \mathrm{HPO}_{4}, 0.2 \mathrm{M} \mathrm{KH}_{2} \mathrm{HPO}_{4}\right)$ and immersed in $30 \%$ sucrose in PBS at $4^{\circ} \mathrm{C}$ overnight or up to $3 \mathrm{~d}$. Before embedding, tissue was equilibrated for $1 \mathrm{~h}$ in 50:50 30\% sucrose in PBS: OCT (Tissue-Tek) and then embedded in the equilibration solution and snap frozen with liquid nitrogen. Adult retinal material was crosssectioned and embryonic eyes were sectioned in the coronal plane at 10 $\mu \mathrm{m}$ using a Leica 1850 cryostat. Sections were transferred onto Superfrost Plus coated slides (Fischer Scientific), air dried for $\sim 4 \mathrm{~h}$ at room temperature, and used immediately for immunohistochemistry or in situ hybridization or stored with desiccant at $-20^{\circ} \mathrm{C}$. Wild-type and mutant tissues were always compared on the same slides. To accurately delineate the mutant region of the retina, images shown for each figure are adjacent sections from a representative animal unless specified otherwise, and the region of Sufu deletion in each eye was confirmed by in situ hybridization (ISH) on a section from the same series.

Hematoxylin and eosin staining. Slides were rehydrated for $30 \mathrm{~min}$ in $1 \times$ PBS and then stained with hematoxylin (Fischer Scientific) for $\sim 7 \mathrm{~s}$. Tissue was then dehydrated through consecutive 2 min incubations in $50 \%$ ethanol $(\mathrm{EtOH}), 70 \% \mathrm{EtOH}, 80 \% \mathrm{EtOH}, 90 \% \mathrm{EtOH} \times 2,100 \%$ $\mathrm{EtOH} \times 2$. Tissue was then stained with eosin (Fischer Scientific) for 1 min and dehydrated using the same protocol as for hematoxylin. Slides were washed $1 \times 2 \mathrm{~min}$ in xylene (Fischer Scientific) and mounted using PerMount (Fischer Scientific). Slides were visualized using an Axioplan microscope and images were captured using an AxioVision camera 2.05 (Zeiss).

Immunohistochemistry/immunocytochemistry and nuclear labeling and TUNEL staining. Immunohistochemistry (IHC) and immunocytochemistry (ICC) were performed as described previously (Wall et al., 2009; Wang et al., 2005). The primary antibodies used in this study were as follows: $\alpha$ Brn3b (Santa Cruz Biotechnology), $\alpha$ ChAT, $\alpha$ Gly-T1, $\alpha \mathrm{TH} 2$, $\alpha \operatorname{Prox} 1$ (Millipore Bioscience Research Reagents), $\alpha$ Calretinin (Swant), $\alpha \mathrm{Vsx} 2$ (a gift from Dr. Rod Bremner, University of Toronto), $\alpha$ CRALBP (a gift from Dr. Jack Saari, University of Washington, Seattle, WA), $\alpha \mathrm{GABA}, \alpha \mathrm{HPC1}$ (Sigma), $\alpha$ GFP (Invitrogen), $\alpha$ Isl1 (Abcam), $\alpha$ Lim1/2 (4F2) (Developmental Studies Hybridoma Bank), $\alpha$ Rhodopsin (B630) (Developmental Studies Hybridoma Bank), $\alpha$ BrdU, $\alpha$ Ki67 (BD Bioscience), and $\alpha$ GAD65 (Abcam). Secondary antibodies used were as follows: $\alpha$ Rabbit FITC (Invitrogen), $\alpha$ Rabbit Cy3, $\alpha$ Mouse Cy3 (Jackson ImmunoResearch), biotinylated $\alpha$ Goat, and Cy3-Streptavidin (GE Healthcare). Nuclei were counterstained with bisbenzimide (DAPI) (Invitrogen) and mounted with fluorescent mounting medium (Dako). Terminal uridine deoxynucleotidyl transferase dUTP nick end labeling (TUNEL) assay was performed using the In Situ Death Detection Kit (Roche Diagnostics) according to the manufacturer's instructions. Nuclei were counterstained with DAPI as above and mounted with fluorescent mounting medium (DAKO). Staining was visualized using a Zeiss fluorescent upright microscope and images were captured using an AxioVision 6.05 camera (Zeiss).

In situ hybridization. ISH was performed as described previously (Jensen and Wallace, 1997) using digoxigenin (DIG)-labeled antisense RNA riboprobes prepared by in vitro transcription from linearized plasmids containing complete or partial cDNA sequences of the following mouse genes: Sufu, Crx, FoxN4, Gli1, Math5, Pax6, Rax, and Vsx2. Sections were analyzed on an Axioplan microscope and digital images were captured using an AxioVision camera 2.05 (Zeiss).

Fluorescent activated cell sorting. Single cell dissociates of trypsinized retinal explants were resuspended in $2 \mathrm{ml}$ of $10 \%$ FBS/DMEM and passed through a $50 \mu \mathrm{m}$ mesh (Millipore). Cells were then placed on ice and sorted using a Dako Cytomation MOFLO (Dako) by StemCore. Sorting parameters gated out dead cells using forward and side scatter and sorted for GFP + cells. The apparatus was cleaned beforehand using $10 \%$ bleach and DEPC water. Cells were collected on ice in $2 \mathrm{ml}$ of $10 \% \mathrm{FBS} / \mathrm{DMEM}$.

$m R N A$ isolation and real-time quantitative PCR analysis of gene expression. mRNA from FACS-sorted cells and dissected embryonic retinas was extracted using Tri-Reagent (Invitrogen) according to manufacturer's instructions. mRNA purity and concentration was determined using spectroscopy (Ependorph BioPhotometer). cDNA was then prepared using Moloney murine leukemia virus (Invitrogen) according to manufacturer's instructions. 


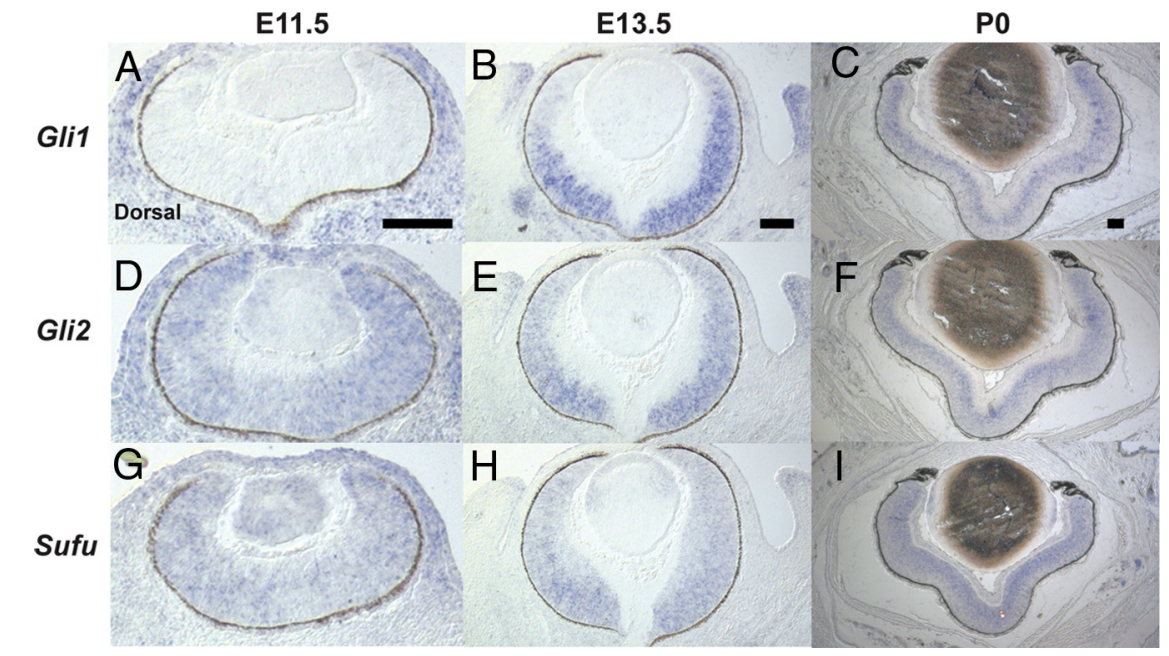

Figure 1. Sufu antagonizes Hh signaling in RPCS. A-I, In situ hybridization on serial coronal sections showing Gli1 (A-C), Gli2 (D-F), and Sufu (G-I) expression in the neuroblast layer atE11.5, E13.5, and P0. Sections are oriented with dorsal on the left. Scale bars, $100 \mu \mathrm{m}$.

For quantitative PCR (qPCR), $2 \mu \mathrm{l}$ of cDNA, $1 \mu \mathrm{l}$ of $10 \mu \mathrm{M}$ forward/10 $\mu \mathrm{M}$ reverse primers, and $10 \mu \mathrm{l}$ of HPLC grade water were added to $15 \mu \mathrm{l}$ of SYBR-Green qPCR master-mix reagent (Sigma). Reactions were performed using an $\mathrm{Mx} 3000 \mathrm{P}$ thermocycler (Stratagene). The primer sequences used were as follows: CRX: F-CAGGTCTGGTTCAAGAATCG, R-ACGAGCCTTGGTCTGTGC; Gli1: F-CAAAAGGGCAGACCAGAAAG, R-TCAATCCAATGACTCCACCA; FoxN4: F-AGCCACACCCCAAACACTAC, R-AAGCTGCCTGTTTTGCTGTT; GAPDH: F-5'TGAAGGGGTCGTTGATGG3, R-5'AAAATGGTGAAGGTCGGTGT3'; Math3: F-CCATCTCAAGCCTCAACCAT, R-CTAATGCTCAGGGGTGGTGT; Math5: F-ATGGCGCTCAGCTACATCAT, R-GGTCTACCTGGAGCCTAGCA; Pax6: F-CGGCAGAAGATCGTAGAGC, R -TGGATACGTTTTCATTGTCCAG; Ptch1: F-TAGCCCTGTGGTTCTTGTCC, R-TGTGGTCATCCTGATTGCAT; Rax: F-TTCATGGACGACACTTCCAG, R-GTTAACCTACCCGAGGTTCG; Sox2: F-ACCTCTTCCTCCCACTCCAG, R-CTGGGCCATGTGCAGTCTAC; Sufu: F-ATTCAGCCCAACAGTGGAAC, R-CCGTCTGTCTAATGCCTTT. Transcript levels were normalized to GAPDH and fold change was calculated using $2^{\mathrm{ddCt}}$. Primer specificity was confirmed by verifying PCR product sizes using electrophoresis and by sequencing PCR products (StemCore) after ligation into pGEM-T plasmid (Promega) as per manufacturer's instructions.

Luciferase activity. Luciferase activity was assayed using the Dual Luciferase Kit (Promega) according to manufacturer's instructions and quantified using a Lumat LB 9507 luminometer (Eg\&G Berthhold). Briefly, explants were immersed in $500 \mu \mathrm{l}$ of $1 \times$ passive lysis buffer solution (Promega), homogenized briefly, and rocked at room temperature for $30 \mathrm{~min}$. The lysate was added to $200 \mu \mathrm{l}$ of luciferin (Promega), luciferase activity was measured, and then $200 \mu \mathrm{l}$ of Stop and Glo solution (Promega) was added to measure Renilla activity.

Image processing, data analysis, and statistics. Images were processed using Adobe Photoshop CS3. Images of control and mutant retinae were taken at the same exposure for each magnification. In the event that contrast/levels were adjusted, they were adjusted for the entire image, and images of both control and mutant retinae were adjusted equally. All scale bars measure $100 \mu \mathrm{m}$. For the in vivo analysis, at least three mutant mice from each age group were examined and compared with wild-type littermates. When counting dissociated cells, at least 150 transfected, GFP + cells were scored per retinal explant. Cell quantification in embryonic retinal sections was performed by counting all the Dapi, Ki67, and BrdU-labeled cells in the ventral and dorsal retina in a region measuring $118 \mu \mathrm{m}$ in length (along the proximal-distal axis of the retina) from three sections/retina at the level of the optic nerve head from a minimum of four eyes/genotype. The position of the Sufu knock-out (KO) region for counting was confirmed by ISH for Sufu on a serial section from the same retina. Staining was visualized as mentioned above for IHC. All data are presented as mean \pm SEM. Statistical significance was evaluated using a two-tailed, unpaired Student's $t$ test and $p<0.05$ was considered statistically significant.

\section{Results \\ Sufu antagonizes Hh signaling downstream of Smo in RPCs}

Previously, we showed that the induction of Shh expression and $H h$ pathway activation in the developing mouse retina follows the central to peripheral wave of GC differentiation (Wang et al., 2005). Therefore, at early stages in retinal development, Hh activation is suppressed in RPCs that are located peripheral to the wave of GC differentiation. Although the expression patterns of Gli1 and Gli2 in the developing retina have been characterized (Wang et al., 2005; Furimsky and Wallace, 2006), we sought to compare the expression domains of these Hh pathway components with the pattern of Sufu expression. At E11.5, a stage before the development of Shh-expressing ganglion cells (Wang et al., 2005), the expression of Gli1, which is a Hh target gene (Lee et al., 1997), is undetectable in the retina (Fig. 1A). In contrast, both Gli2 and Sufu are expressed throughout the neuroblast layer at this stage (Fig. 1D,G). At E13.5, the region of Gli1 expression does not extend to the peripheral-most part of the retina (Fig. $1 B$ ), while both Gli2 and Sufu continue to be expressed throughout the neuroblast layer (Fig. $1 E, H$ ). In the P0 retina, Gli1, Gli2, and Sufu are all coexpressed in the neuroblast layer throughout the retina (Fig. 1C,F,I). Thus, the expression of Sufu and Gli2 in RPCs precedes Hh pathway activation in the retina and expression is maintained in the region of active Hh signaling.

To determine whether Sufu can functionally antagonize Smo signaling in RPCs, we investigated the effect of increased Sufu expression on Hh pathway activation in retinal explants. Coelectroporating explants with an expression vector for Smo-M2, a constitutively active form of Smo (Xie et al., 1998) that cellautonomously activates the Hh pathway in RPCs (Yu et al., 2006; Wall et al., 2009) and a Gli-Luciferase reporter (Gli-Luc) (Sasaki et al., 1997) resulted in a significant increase in Gli-Luc activity compared with controls, and this effect was attenuated approximately threefold when explants were coelectroporated with a Sufu expression vector (Ding et al., 1999). (supplemental Fig. 1 A, available at www.jneurosci.org as supplemental material). We next asked whether conditional inactivation of Sufu in RPCs was sufficient to activate the Hh pathway. Electroporating explants from $S u f u^{\mathrm{F} / \mathrm{F}}$ mice with an expression vector for Cre recombinase reduced wild-type levels of Sufu after 2.5 days in vitro (DIV) and 4 DIV in the transfected population (supplemental Fig. $1 B$, available at www.jneurosci.org as supplemental material) and increased Gliluciferase activity by fivefold and 16-fold after 2.5 and 5 DIV, respectively (supplemental Fig. 1C, available at www.jneurosci.org as supplemental material). Thus, Sufu inhibits Hh signaling in RPCs, which is consistent with its previously reported antagonistic function on the Hh pathway (Ruel and Thérond, 2009).

\section{Sufu is required in vivo to antagonize Hh signaling in RPCs}

To study the functions of Sufu in vivo, we generated a retinaspecific conditional knock-out (CKO) of Sufu by crossing Sufu $u^{F / F}$ mice with a transgenic line that expresses Cre recombinase under 
E11.5
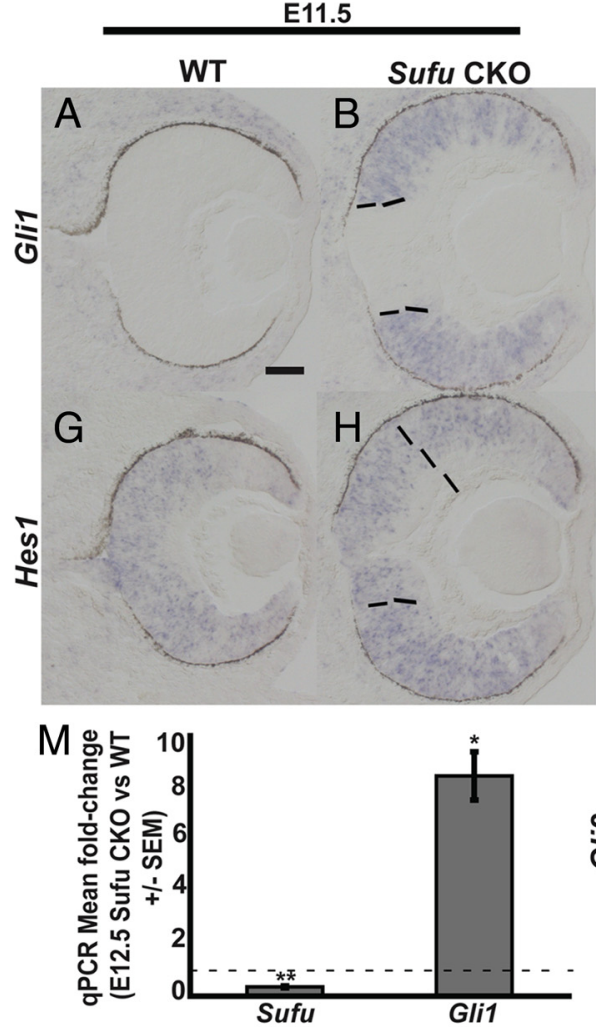

E13.5
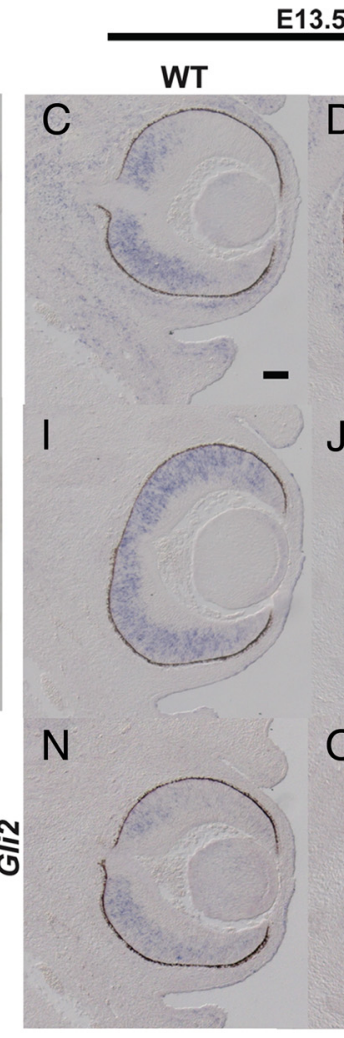

WT

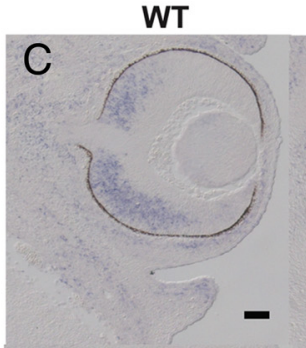

I

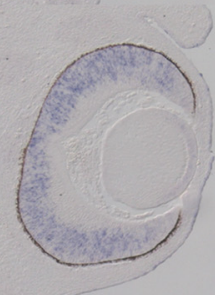

J

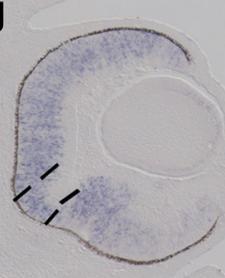

O

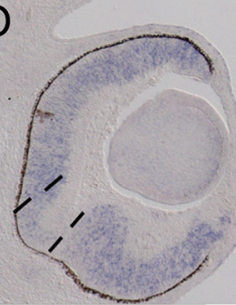

Sufu CKO
E15.5
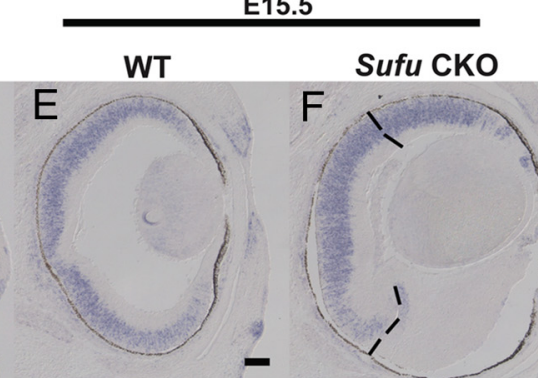

K

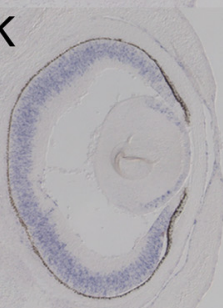

P

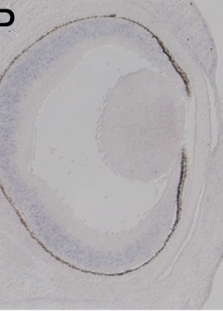

$\mathrm{F}$
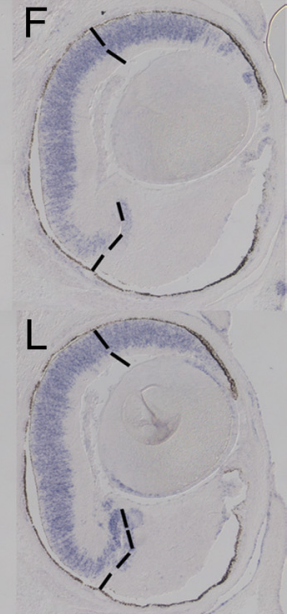

Q

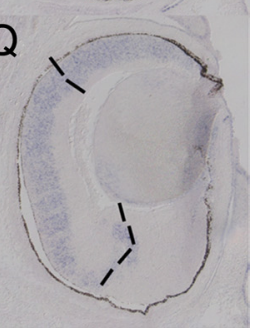

Figure 2. Increased levels of Hh signaling in Sufu CKO retinae. $\boldsymbol{A}-\mathbf{Q}$, In situ hybridization for Gli1 (A-F), Hes 1 ( $\mathbf{G}-\boldsymbol{L}$ ), and Gli2 ( $\mathbf{N}-\mathbf{Q})$ mRNA in control and mutant retinae at E11.5, E13.5, and E15.5. Dorsal is up. Note the expanded domain of Gli1 expression in the Sufu CKO retina. The dashed lines indicate the border of the mutant region in the peripheral ventral retina, which was determined by ISH for Sufu on a serial section from the same eye. Because of the sectorial gaps in Cre activity in this line, not all retinal sections will have Sufu deletion in the dorsal retina. Scale bars, $100 \mu \mathrm{m}$. M, Mean fold-changes of Sufu and Gli1 cDNA levels in whole Sufu CKO retinae compared with whole WT retinae at E12.5 normalized to GAPDH. Error bars represent SEM. ${ }^{*} p<0.05,{ }^{* *} p<0.005$.

the control of the $\alpha \mathrm{P} 0$ element of the Pax6 promoter $(\alpha-C r e)$, which induces Cre expression in RPCs of the peripheral retina beginning at $\sim$ E10.5 (Marquardt et al., 2001) (supplemental Fig. $2 A$, available at www.jneurosci.org as supplemental material). For simplicity, $\mathrm{Cre}^{+} ; \mathrm{Sufu}^{\mathrm{F} / \mathrm{F}}$ mice will be referred to as $\mathrm{Sufu} \mathrm{CKO}$ mice, and control $\mathrm{Cre}^{-}$; $\mathrm{Sufu}{ }^{F / F}$ mice will be referred to as wild type (WT). As we were unable to detect any overt differences in morphology or gene expression when comparing $\mathrm{Cre}^{+}$; Sufu ${ }^{+/ F}$ and WT mice (data not shown), we restricted our analysis to WT and Sufu CKO mice. The $\alpha$-Cre line exhibits a gap in crerecombinase activity in the dorsal-temporal quadrant of the retina (Xu et al., 2007), such that Gfp expression (from the $\alpha$-Cre transgene) (supplemental Fig. $2 B$, available at www.jneurosci.org as supplemental material) and Sufu downregulation are more prominent in the ventral compared with the dorsal retina in coronal sections from the temporal side of the eye (supplemental Fig. $2 B$, compare $E, F$, available at www.jneurosci.org as supplemental material). Therefore, because of the consistency of Cre activity across the ventral region of the retina, we have focused primarily on the phenotype in the ventral retina, and the region of Sufu deletion in the embryonic Sufu CKO retinas was confirmed by ISH for Sufu on serial sections.

To determine whether Sufu deletion in the embryonic retina resulted in Hh pathway activation, we examined the expression of Gli1. At E11.5 and E13.5, the domain of Gli1 expression was expanded in the ventral and dorsal regions of the Sufu CKO retinae compared with WT (Fig. $2 B, D$ ). The increase in Gli1 expression was confirmed by reverse transcription (RT)-qPCR analysis of whole mutant retinae at E12.5 (Fig. $2 \mathrm{M}$ ). In contrast, the ex- pression of Hes 1 , which is regulated by Hh during later stages of development (Wall et al., 2009), was expressed in the mutant retinae at E11.5 (Fig. $2 H$ ) but appeared to be reduced in the ventral region of the mutant retina at E13.5 (Fig. $2 J$ ). Gli2 was expressed throughout the mutant regions of the retina at E13.5 (Fig. 2O). However, by E15.5, the expression of Gli1, Hes1, and Gli2 was undetectable in the ventral region of the mutant retina (Fig. $2 F, L, Q$ ) and the expression of Gli1 was patchy in the dorsal retina (Fig. $2 F$ ). Therefore, consistent with the effect observed in explant culture, genetic elimination of Sufu in RPCs in vivo results in ectopic Hh pathway activation, although this activation in the ventral retina appears to be transient in vivo as Hh signaling is not sustained in the $S u f u$-null region of the retina at later gestational and postnatal stages (data not shown).

Sufu deletion resulted in the formation of a prominent cellular mass in the peripheral ventral retina that was visible by E12.5 and that persisted in the adult eye (Fig. 3A-H) (data not shown). By contrast, the dorsal adult retina did not exhibit hyperplasia and appeared thinner and disorganized (Fig. $3 F$ ). Whole eyes of mutant mice were frequently larger than those of WT littermates (Fig. 3I). The $\alpha$-Cre transgene is also expressed in amacrine cells in the central retina (Marquardt et al., 2001). The central retina of P0 Sufu CKO mice appeared relatively normal, with well demarcated ganglion and neuroblast layers, and the expression of the $\alpha$-Cre transgene, marked by Gfp, in amacrine cells was readily apparent (supplemental Fig. $3 A-K$, available at www.jneurosci. org as supplemental material). By P21, the retina located outside the peripheral mass in the mutants exhibited extensive abnormalities that included rosettes, folds, and disrupted lamination 


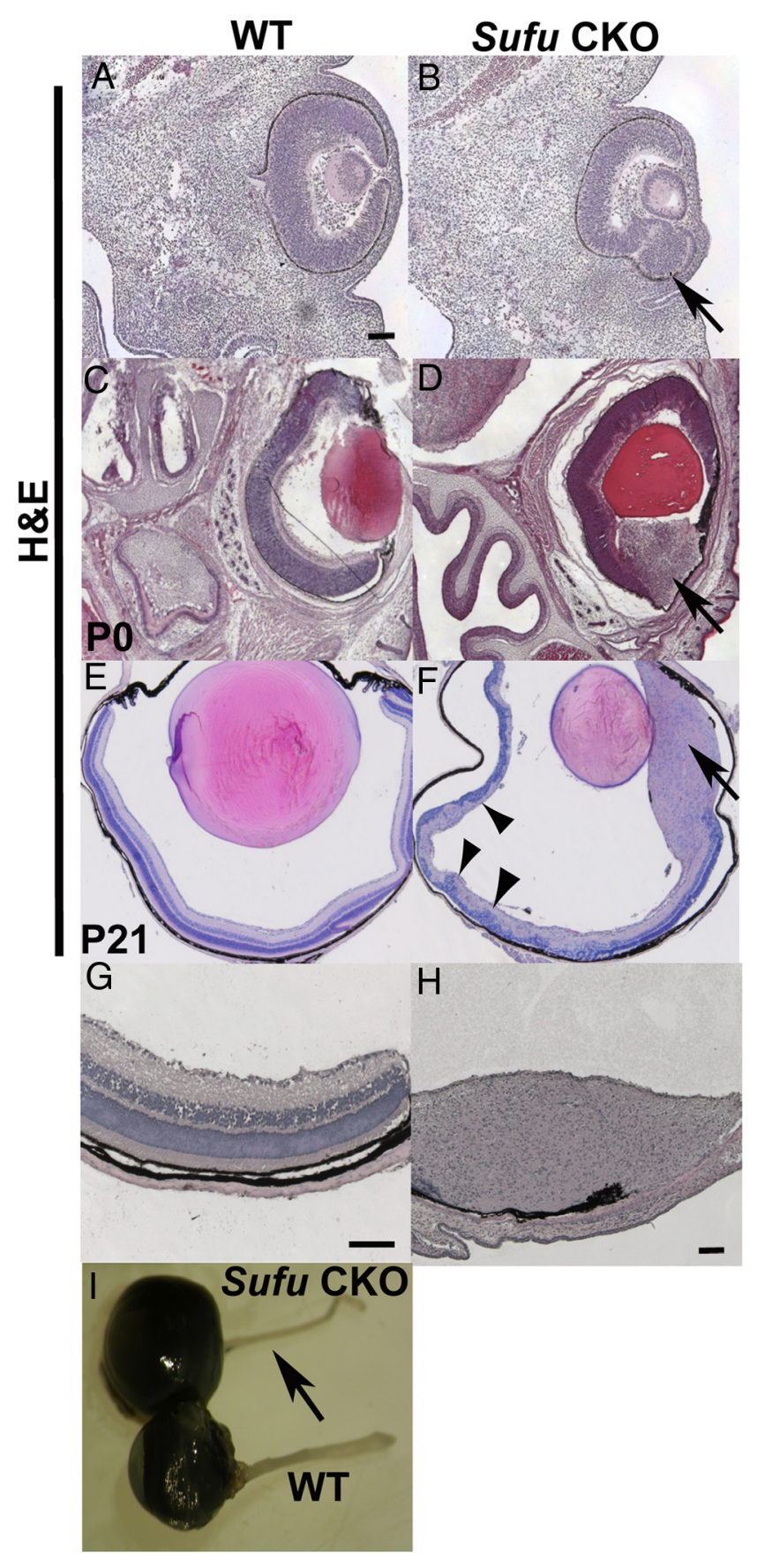

Figure 3. The retinae of Sufu CKO mice display severe morphological defects. $\boldsymbol{A}-\boldsymbol{H}$, Hematoxylin and eosin (H\&E) staining of wild-type and mutant retinas at E12.5 $(\boldsymbol{A}, \boldsymbol{B}), \mathrm{PO}(\boldsymbol{C}, \boldsymbol{D}), \mathrm{P} 21$ $(\boldsymbol{E}, \boldsymbol{F})$, and $\mathrm{P} 18(\boldsymbol{G}, \boldsymbol{H})$. Note the formation of a cellular mass in the ventral peripheral retina in $\boldsymbol{B}$, $\boldsymbol{D}, \boldsymbol{F}$ (arrow). At P21, the remaining retina appears thinner with areas of abnormal lamination $(\boldsymbol{F}$, arrowheads) $(\boldsymbol{H})$. High-magnification view of the ventral mass of the mutant eye at P18 reveals absence of lamination in this region $(I)$. Scale bars, $100 \mu \mathrm{m}$. $I$, Dissected eyes and optic nerves from a WT and Sufu CKO mouse at P18. Note that the mutant eye is larger than the WT eye, and the optic nerve of the mutant eye is notably thinner (arrow).

(Fig. 3F; supplemental Fig. $3 L$, available at www.jneurosci.org as supplemental material). Whether these central retinal abnormalities are a direct consequence of $S u f u$ deletion in amacrine cells or are secondary to the hyperplasia in the peripheral retina is difficult to determine, and for this reason we restricted our analysis to the phenotype associated with Sufu deletion in the peripheral retina.
Sufu-null progenitors differentiate exclusively into horizontal and restricted classes of amacrine interneurons

To identify the types of differentiated cells in the Sufu CKO ventral retina, we performed IHC for lineage-specific markers. At P0, the $S u f u$-null region of the retina can be identified based on the distinct morphological characteristics as well as by the expression of the ROSA- $\beta$-Gal cre reporter, which overlaps completely with the hyperplastic region in the ventral retina (supplemental Fig. $4 A, B$, available at www.jneurosci.org as supplemental material). At P0, we found that expression of syntaxin, which marks amacrine and horizontal cells, was expanded in the ventral retina (supplemental Fig. 4C,D, available at www.jneurosci.org as supplemental material), suggesting that the cells differentiated as interneurons. Cells within the Sufu-null region of the retina also expressed the amacrine/GC marker Isl1 (supplemental Fig. $4 E-$ $F^{\prime}$, available at www.jneurosci.org as supplemental material) and the horizontal interneuron marker Lim1/2 (supplemental Fig. $4 G-H^{\prime}$, available at www.jneurosci.org as supplemental material). Because several of the cell-type specific markers are not expressed until late in development, we conducted further analysis of P18-P21 retinae, a stage when retinal histogenesis is complete. The mutant region of the adult Sufu CKO retina was identified morphologically, based on the increase in thickness and abnormal spacing of the cells that was a consistent feature of the peripheral mutant retina at this stage. Consistent with our analysis at P0, the majority of the cells in the Sufu-null region of the retina at P18 expressed syntaxin (Fig. 4B,C). Significant numbers of cells stained for the amacrine marker calretinin (Fig. $4 E, F)$, although at lower intensity compared with the WT retina (Fig. 4D). A subset of cells also expressed the amacrine/horizontal/bipolar marker Proxl (Fig. 4H,I) and the amacrine/horizontal marker calbindin (data not shown). In contrast, very few, if any, of the cells in the mutant region expressed markers exclusive to other differentiated cell types, including GCs (data not shown), bipolar cells, photoreceptors or Müller glia (supplemental Fig. 5A-H, available at www.jneurosci.org as supplemental material). Amacrine cells are extremely diverse, and can be subclassed on the basis of their expression of neurotransmitters or proteins involved in neurotransmitter metabolism (NguyenLegros et al., 1997; Demb, 2007). Some interneurons in the Sufunull region were dopaminergic, based on the expression of tyrosine hydroxylase (TH2) (Fig. $4 K, L$ ). In contrast, very few, if any, of the cells within this region were glycinergic or cholinergic, based on the absence of glycine transporter 1 (Gly-T1) (Fig. $4 N, O$ ) or choline acetyltransferase (ChAT) (Fig. 4S,T), respectively. Although we detected expression of GAD65, which converts glutamate to GABA, within the mutant region (Fig. $4 W, X$ ), the expression of GABA (Fig. 4Q,R) was weak to undetectable, suggesting that GABAergic differentiation was aberrant. Our results suggest that Sufu deletion leads to a loss of cell diversity and nearly all cells in the Sufu-null region of the retina exhibit an interneuron phenotype. Importantly, although we observed a consistent phenotype in the mutant region of the adult Sufu CKO retinae, we cannot rule out the possibility that the effects observed at or near the WT-mutant border occur due to non-cell autonomous processes, such as cell mixing or disruption of the physical architecture of the retina.

\section{$S u f u$ is required to maintain RPC multipotency}

To gain insight into the developmental origin of the postnatal Sufu CKO phenotype, we addressed whether the effect of Sufu deletion in the ventral retina was associated with perturbed expression of transcription factors that regulate RPC proliferation, 

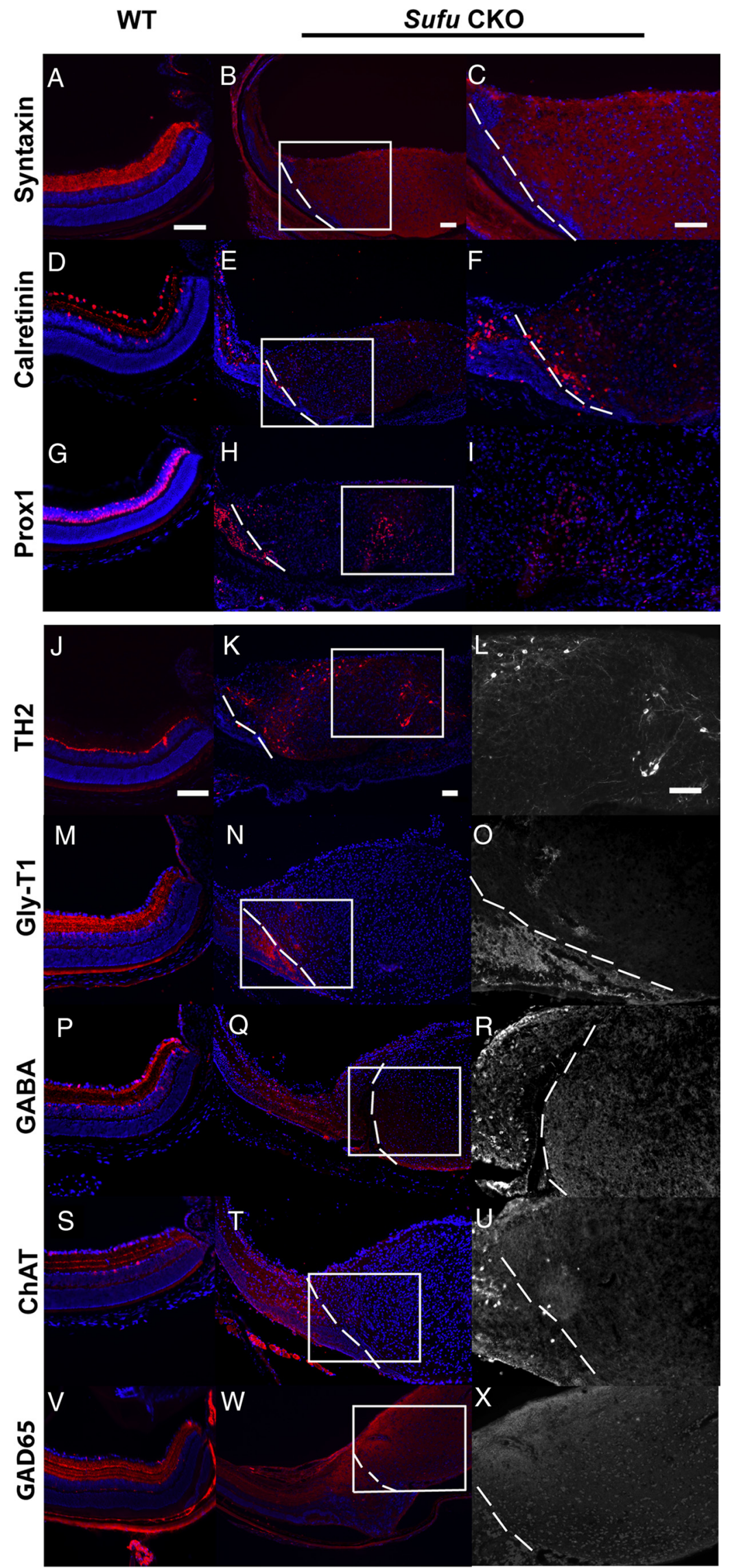

Figure 4. Cells in the peripheral retina of Sufu CKO mice differentiate into a restricted subset of interneurons. $A-X$, Fluorescent immunohistochemistry for interneuron markers syntaxin $(A-C)$, calretinin $(D-F)$, Prox1 (G-I), TH2 (J-L), Gly-T1 (M-O), GABA neurogenesis, and multipotency at earlier stages in development. Cells in the embryonic Sufu CKO ventral retina expressed Sox2, consistent with their neural precursor status (Fig. $5 B, D$ ). Unexpectedly, however, Sufu deletion was associated with the dysregulation of transcription factors that are required to maintain RPC function. The expression of Pax6, Rax, and $V s \times 2$ was downregulated in the Sufu CKO peripheral retina at E12.5 and E14.5 (Fig. 5E-P). Quantification of transcript levels in whole mutant eyes confirmed the reduction in Pax6 and Rax expression at E12.5 and E14.5 and an upregulation of Sox2 expression (Fig. 5Q). Based on the requirement for Pax6 in the maintenance of RPC multipotentiality (Marquardt et al., 2001; Oron-Karni et al., 2008), the downregulation of Pax6 in the Sufu-null region of the retina suggested that RPC multipotency is compromised.

To investigate the consequences of Sufu deletion on cell-fate diversification, we monitored the expression of transcription factors that are involved in lineage determination or that are expressed in postmitotic lineage-committed precursor cells. Math5, which is required to specify GC fate (Brown et al., 2001; Wang et al., 2001), was downregulated in Sufu-null region of the ventral retina at E12.5 and E14.5 (Fig. 6A-D). Consistent with the loss of Math 5 expression, we did not detect GC differentiation in the Sufu-null region of the ventral retina during embryogenesis (Fig. 6E-H) or at P0 (supplemental Fig. $4 I-J^{\prime}$, available at www. jneurosci.org as supplemental material), indicating that GC differentiation in this region was abrogated, rather than delayed. Similarly, the expression of Foxn4 and Crx, which are required for amacrine/ horizontal cell (Li et al., 2004) and rod/cone photoreceptor development (Furukawa et al., 1999), respectively, was also undetectable in the Sufu-null region of the ventral retina at E12.5 and E14.5 (Fig. 6I-O) (data not shown). Consistent with the loss of Foxn4 expression, the expression of its downstream targets, Math3 and NeuroD1 (Li et al., 2004), was also undetectable in the Sufu-null region (Fig. 6O) (data not

$(\boldsymbol{P}-\boldsymbol{R})$, ChAT $(\boldsymbol{S}-\boldsymbol{U})$, and GAD65 $(\boldsymbol{V}-\boldsymbol{X})$ in the retinas of 3-week-old wild-type and Sufu CKO mice. Nuclei are counterstained with DAPI. $\boldsymbol{C}, \boldsymbol{F}, \boldsymbol{I}, \boldsymbol{L}, \boldsymbol{O}, \boldsymbol{R}$, and $\boldsymbol{U}$ are high-magnification views of the boxed region in the images to the left of those panels. Dashed lines in $\boldsymbol{C}, \boldsymbol{F}, \boldsymbol{O}, \boldsymbol{R}, \boldsymbol{U}$, and $\boldsymbol{X}$ delineate the border between WT (left) and mutant tissue (right) in the Sufu CKO retina to provide comparison of the immunofluorescent signal in the WT and mutant regions of the Sufu CKO retinae. Scale bars, $100 \mu \mathrm{m}$. 
E12.5

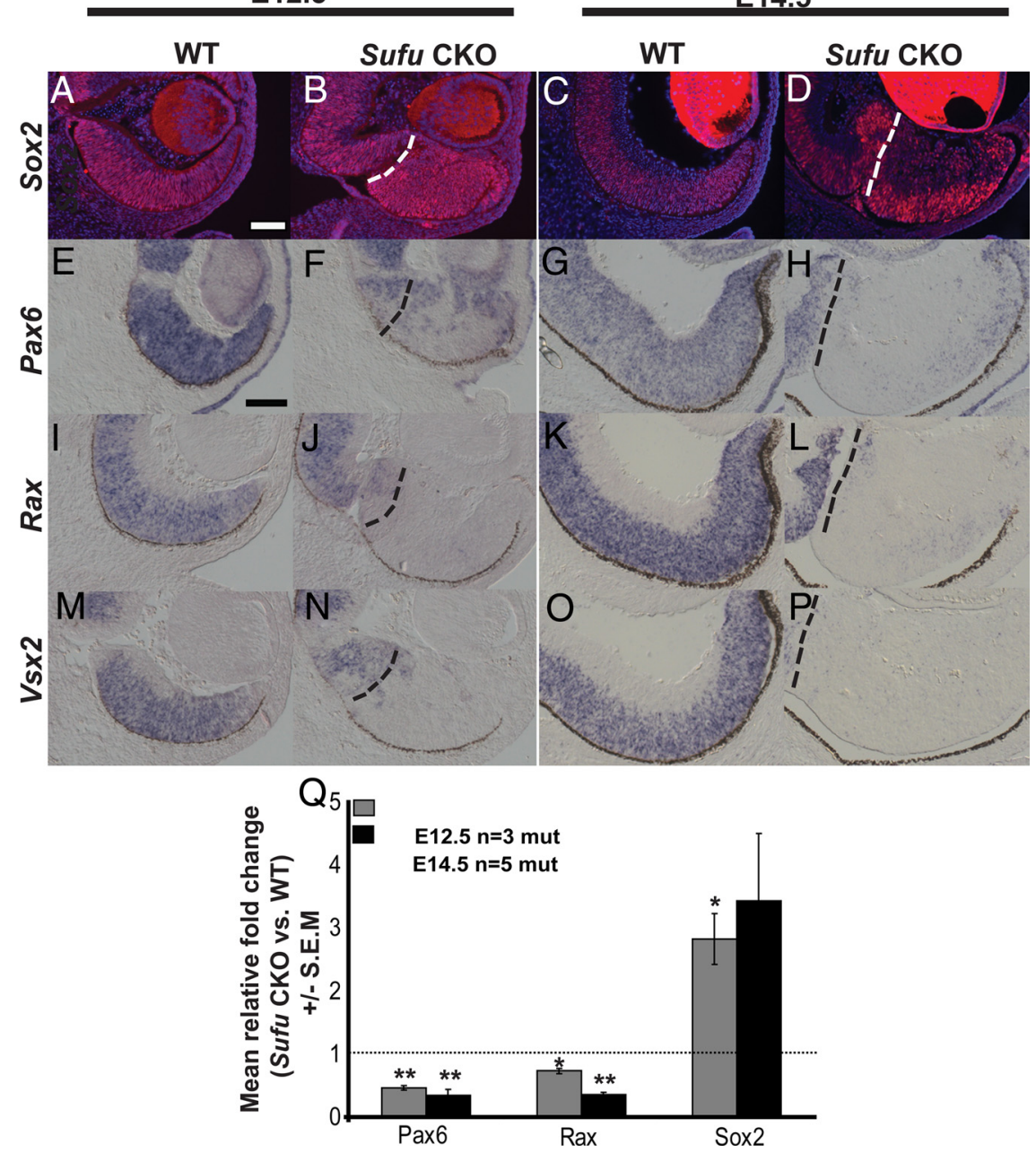

Figure 5. Cells in the peripheral ventral retina of Sufu CKO mice exhibit aberrant expression of transcription factors required to maintain the multipotent and proliferative capacity of RPCS. $\boldsymbol{A}-\boldsymbol{P}$, Fluorescent immunohistochemistry for Sox2 (A-D) and in situ hybridization for Pax $6(\boldsymbol{E}-\boldsymbol{H}), \operatorname{Rax}(\boldsymbol{I}-\boldsymbol{L})$, and $V s \times 2(\boldsymbol{M}-\boldsymbol{P})$ in the retinae of wild-type and mutant (mut) mice at E12.5 and E14.5. Dashed lines delineate the border of the mutant region in the peripheral ventral retina. Note the downregulation or absence of Pax6, Rax, and Vsx2 in the peripheral retinae of Sufu CK0 mice. Scale bars, $100 \mu \mathrm{m}$. Q, RT-qPCR was used to determine mean fold-changes of Pax6, Rax, and Sox2 CDNA levels in whole Sufu CKO retinae compared with whole WT retinae at E12.5 and E14.5 normalized to GAPDH. Error bars represent SEM. ${ }^{*} p<0.05,{ }^{* *} p<0.005$.

shown). The absence of Rax and Vsx2 expression, which are required for Müller glia (Furukawa et al., 2000) and bipolar cell (Burmeister et al., 1996; Livne-Bar et al., 2006) development, respectively, was also consistent with the absence of these differentiated cell types at later stages in the Sufu KO retina. The downregulation of these transcription factors in E14.5 Sufu CKO retinae was confirmed with RT-qPCR (Fig. 6O). In contrast to the ventral retina, in retinal sections where there was clear deletion of Sufu in the dorsal retina, we observed a consistently weak reduction of Math5 (supplemental Fig. 2, compare $H$, $J$, available at www.jneurosci.org as supplemental material) (data not shown) FoxN4, NeuroD, and Crx (data not shown) expression.

The dramatic changes in gene expression in Sufu-null cells were not associated with altered cell survival, as we did not detect an increase in TUNEL-positive cells in the ventral portion of the retina at E12.5, E14.5, or E15.5 (data not shown). We detected normal expression patterns of $\operatorname{Pax} 2$ and $O t x 1$, which are markers of optic stalk and ciliary margin, respectively (supplemental Fig. 6, available at www.jneurosci.org as supplemental material), indicating that cells in the mutant region had not transdifferenti- ated into other ocular tissue types. Therefore, in the absence of $S u f u$, ventral RPCs show aberrant expression of transcription factors that are required to maintain RPC multipotency and identity, and fail to initiate the expression of transcription factors for the specification and/or differentiation of all seven retinal cell lineages. Our data demonstrate a novel role for Sufu in the maintenance of RPC multipotency during early retinal development.

\section{Sufu-null cells prematurely exit the cell cycle}

We next sought to characterize the effect of Sufu on RPC proliferation. Acute Sufu deletion in perinatal retinal explants increased proliferation nearly threefold (Fig. 7A), indicating that Sufu is a potent negative regulator of RPC proliferation. However, quantitative analysis of the cycle kinetics in vivo revealed that the in vivo effects of Sufu loss on RPC proliferation are complex. In vivo, the Sufu-null region of the ventral, and to a lesser extent the dorsal, retina is hyperplastic (Fig. $7 \mathrm{~L}$ ). At E11.5, Sufu loss in both regions is associated with a significant increase in the BrdU labeling index (Fig. $7 L$ ), indicative of a faster cell cycle at this stage. By E13.5, however, we noted differences in cellcycle kinetics between the ventral and dorsal RPCs. Sufu loss in ventral RPCs resulted in a slowing of the cell cycle and premature cell-cycle exit, as shown by the reduction in $\mathrm{Ki67}+$ cells beginning at E14.5 (Fig. 7C-E,L). By E15.5 and onwards there were no cycling cells present in the ventral retina (Fig. $7 G$ ) (data not shown). The reduction in cell-cycle rate and premature cell-cycle exit was associated with ectopic expression of the cyclindependent kinase inhibitor $p 57^{K i p 2}$ in the Sufu-null region of the ventral retina at E12.5 and E14.5 (Fig. $7 I, K$ ) and a reduction in the anti-neurogenic factor, Hes 1 expression (Fig. $2 D, J$ ). In contrast, dorsal RPCs did not undergo premature cell-cycle exit (Fig. $7 L$ ). Thus, initially, dorsal and ventral RPCs respond to Sufu loss by faster cycling, which is followed by a slowing of the cell cycle and in the case of ventral progenitors, premature cellcycle exit.

\section{Aberrant Hh activity and loss of RPC identity in Sufu CKO retinae is mediated by Gli2}

Sufu binds all three Gli proteins and has recently been shown to be an important regulator of Gli2 and Gli3 processing (Humke et al., 2010; Wang et al., 2010). However, the epistatic relationship between Sufu and Gli2 in vivo is unknown. As Gli2 is the primary activator of Hh signaling in vivo (Bai et al., 2002) and is required for full Hh pathway activation in RPCs (Wall et al., 2009), we sought to determine whether genetic elimination of Gli2 could rescue the effects of Sufu deletion in RPCs. In retinal explants, genetic elimination of Gli2 was sufficient to prevent the increase 
in Gli-Luc activity observed after acute Sufu deletion in a dose-dependent manner (Fig. $8 A$ ), indicating that transcriptional activation of the Hh pathway in the context of Sufu deletion is mediated by Gli2.

We next asked whether genetic elimination of Gli2 was sufficient to rescue the effects of conditional Sufu deletion in vivo by generating Sufu CKO;Gli2 ${ }^{-1-}$ (double $\mathrm{KO})$ mutant mice. Because homozygous deletion of Gli2 is lethal at late stages in embryonic development (Mo et al., 1997), the retinae of Sufu CKO; Gli2 ${ }^{-1-}$ mice were examined at E14.5. Gli2 deletion alone is not sufficient to dramatically alter the expression of Sufu (supplemental Fig. 2E, available at www.jneurosci.org as supplemental material), the proneural gene, Math5 (supplemental Fig. 2, available at www.jneurosci.org as supplemental material), or Pax6 and Vsx2 (Furimsky and Wallace, 2006). Remarkably, removal of Gli2 in Sufu CKO retinae restored RPC multipotentiality based on the upregulation of Pax 6 and Rax expression in double KO retinae compared with Sufu CKO retinae (Fig. $8 B-G$ ). In addition, the expression of Foxn4, Math3, Math5, and $\mathrm{Cr} x$ were restored in double $\mathrm{KO}$ retinae (Fig. $8 \mathrm{H}-\mathrm{S}$ ), although not to the levels observed in WT retinae. Together, these data demonstrate that Sufu regulation of Gli2 activity is required for RPC multipotentiality.

\section{Discussion}

Shh signaling to progenitor cells in the developing CNS is required for fate specification, proliferation, and maintenance of the undifferentiated state. Here, we have investigated the requirement for Sufu, a downstream regulator of the Shh response, in RPCs. Our results indicate that Sufu acts downstream of Smo to suppress Gli2 activity and Hh pathway activation. Functionally, Sufu is required to antagonize RPC proliferation and, unexpectedly, it is also required for sustained expression of the retinal determination gene network and the maintenance of multipotentiality of RPCs. Finally, we show that the effect of Sufu on RPC multipotentiality is mediated primarily through Gli2. In summary, Sufu antagonism of Gli2 identifies a novel intrinsic component of RPC multipotentiality.

Consistent with previous studies that have characterized Sufu as a critical regulator of mammalian Hh signaling (Taylor et al., 2002; Cooper et al., 2005; Svard et al., 2006; Varjosalo et al., 2006), we show that conditional deletion of Sufu in RPCs in vitro and in vivo results in aberrant Hh pathway activity, increased proliferation, and hyperplasia. The transient increase of Hh signaling that we observe in the $S u f u$ CKO retina could be caused by a failure to sustain the Gli2 expression and/or activity, which is consistent with labile nature of Gli proteins in Hh-activated cells (Humke et al., 2010) and a requirement for Sufu in promoting Gli stabilization (Chen et al., 2009). Interestingly, proliferation was also not maintained indefinitely in the retinae of $S u f u$ CKO mice, and Sufu-null progenitors in the ventral retina exited the cellcycle en mass between E14.5 and E15.5. There were differences in
E12.5

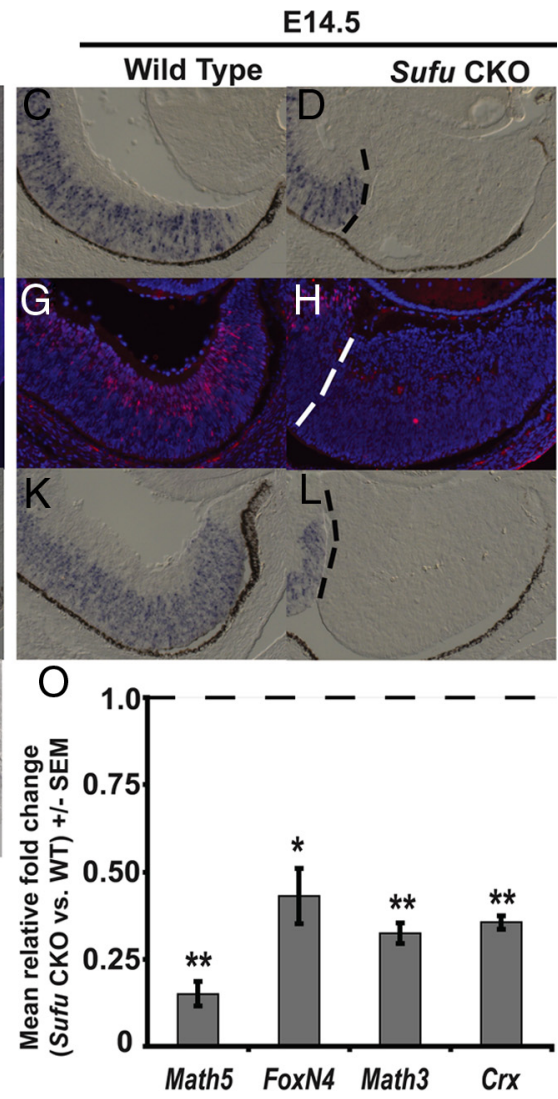

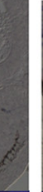

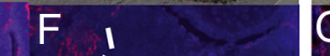

Sufu CKO

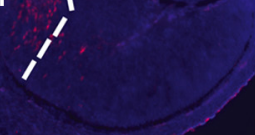

J
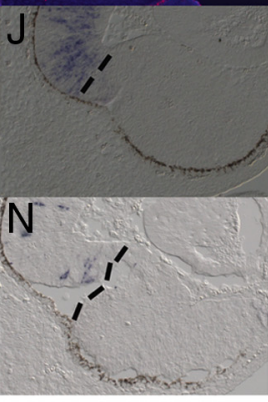

Math5 FoxN4 Math3

Crx

Figure 6. Multipotency is lost in Sufu-null RPCS. $\boldsymbol{A}-\boldsymbol{N}$, In situ hybridization for Math5 (A-D), FoxN4 (I-L), and Crx $(\boldsymbol{M}-\boldsymbol{N})$ and fluorescent immunohistochemistry for Brn3b $(\boldsymbol{E}-\boldsymbol{H})$ with nuclei counterstained with DAPI in WT and Sufu CKO retinae at E12.5 and Foxpression in the peripheral retinae of Sufu CKO mice. Signal in the mutant region in $\boldsymbol{D}$ is nonspecific staining that 列 and Crx levels in whole E14.5 Sufu CKO retinae compared with whole WT retinae normalized to GAPDH. $n=5$ retinae per genotype. Error bars represent SEM. ${ }^{*} p<0.005 ;{ }^{* *} p<0.05$.

the severity of the phenotype between dorsal and ventral Sufu CKO RPC, the latter exhibiting a stronger phenotype. This could reflect dorsal-ventral differences in Sufu function or may be due to secondary effects related to incomplete deletion of Sufu in the dorsal retina.

The precocious cell cycle exit of ventral RPCs in the Sufu CKO mouse may be mediated by three nonmutually exclusive mechanisms. First, it could be secondary to the downregulation of $\mathrm{Hh}$ activation in this model. Second, deregulated expression of cellcycle genes could contribute to early cell-cycle exit in Sufu-null progenitors. Conditional deletion of Sufu in vivo resulted in an increase in the cell-cycle rate followed by ectopic expression of the cell-cycle inhibitor $p 57^{\text {Kip } 2}$ at a stage prior to and during the timing of the downregulation of Ki67 expression. $p 57^{\text {Kip } 2}$ directs cell-cycle exit in a subpopulation of RPCs during retinogenesis (Dyer and Cepko, 2000), and Hh signaling is required for $p 57^{\text {Kip2 }}$ expression and cell-cycle exit in the zebrafish retina (Shkumatava and Neumann, 2005; Locker et al., 2006). While sufficient evidence supports the mitogenic role for Shh in mammalian RPCs (Jensen and Wallace, 1997, Yue et al., 2009, Wall et al., 2009), there is little evidence to suggest a role for Shh in driving cell-cycle exit in mammalian RPCs. Although future studies are required, our data indicate that $H h$ signaling may also control cell-cycle exit by modulating $p 57^{\text {Kip } 2}$ expression in mammalian RPCs, thus providing further evidence of Hh pathway conservation. Third, Sufu regulation of retinal determination genes could also affect RPC 

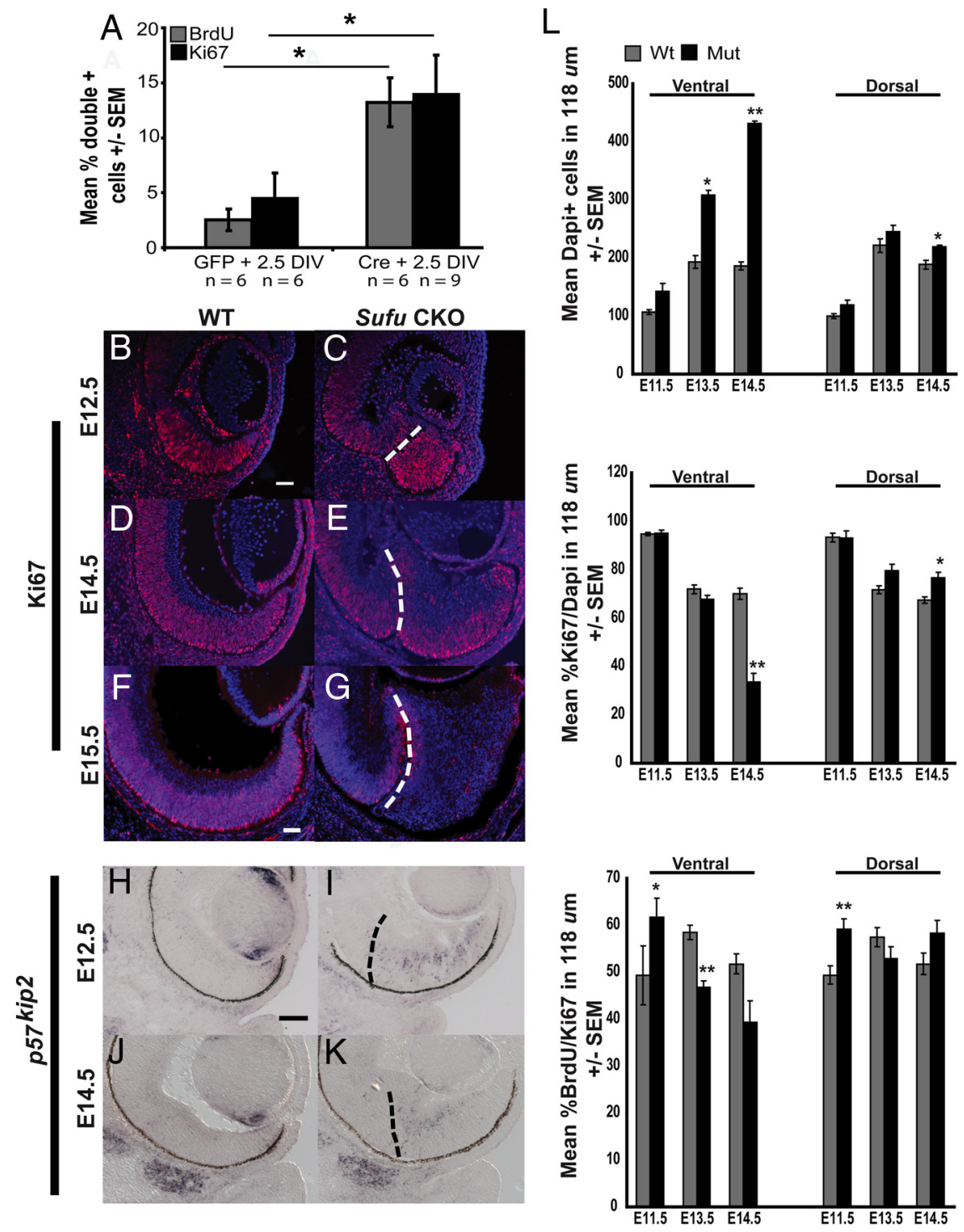

Figure 7. Sufu deletion results in aberrant RPC proliferation. $A$, Acute deletion of Sufu induces proliferation in RPCs. PO Sufu/F retinas were coelectroporated with expression vectors for eGFP and Cre or eGFP and an empty vector and cultured as explants for 2.5 DIV. Explants were then pulsed with BrdU for $5 \mathrm{~h}$, dissociated, and GFP + cells were scored for the expression of Ki67 or BrdU incorporation. $\boldsymbol{B}-\boldsymbol{K}$, Immunostaining for Ki67 (B-G) and in situ hybridization for $p 57^{K i p 2}(\boldsymbol{H}-\boldsymbol{K})$ in the retinae of WT and Sufu CKO mice at the indicated ages. Nuclei are counterstained with DAPI. In the WT retina, RPCs are localized to the neuroblast layer and express Ki67 at E12.5 (B), E14.5 (D), and E15.5 (F). Cells in the mutant region of the retina express Ki67 at E12.5 $(\boldsymbol{C})$ and E14.5 (E). However, few cells in the mutant region were Ki67 + at E15.5 (G). The cell-cycle inhibitor p57(Kip2) is upregulated in the ventral retina of Sufu CKO mice at E12.5 $(\boldsymbol{H}, \boldsymbol{I})$ and E14.5 $(\boldsymbol{J}, \boldsymbol{K})$. Dashed lines delineate the border of the mutant region in the ventral peripheral retina. Scale bars, $100 \mu \mathrm{m}$. L, Quantitative analysis of cell number (DAPI), progenitor cells (\%Ki67+), and BrdU labeling index (\% BrdU + Ki67 + /Ki67+) in the ventral and dorsal retina of wild-type and Sufu CK0 mice atE11.5 (WT, $n=6 ;$ mutant, $n=$ 5 eyes), E13.5 (WT, $n=8$; mutant, $n=7$ eyes), and E14.5 (WT, $n=6$; mutant, $n=4$ eyes). All error bars represent SEM. ${ }^{*} p<$ $0.05 ;{ }^{* *} p<0.005$. mut, Mutant.

proliferation. Proliferation defects are observed in RPCs lacking Pax6 (Marquardt et al., 2001), Rax (Zhang et al., 2000), Vsx2 (Burmeister et al., 1996; Green et al., 2003), and Hes1 (Lee et al., 2005). The marked downregulation of Pax6, Rax, and Vsx2 in concert followed by the loss of Hes 1 may also contribute to the inability of $S u f u$-null cells to remain in cycle indefinitely.

We show that conditional deletion of Sufu resulted in the downregulation of Pax6, which is consistent with the antagonistic relationship between high levels of Shh signaling and Pax6 expression in the neural tube (Ericson et al., 1997). Analysis of mutant retinae at $\mathrm{P} 18$ revealed that $S u f u$-null progenitors were restricted toward an interneuron lineage. This phenotype is consistent with that observed in the retinae of mice with a conditional deletion of Pax6 (Pax6 CKO) (Marquardt et al., 2001). However, several observations suggest that the Sufu CKO phenotype is not exclusively the result of altered Pax6 expression. Sufu CKO RPCs downregulate transcription factors, including Rax, NeuroD, Vsx2, and Crx that continue to be expressed in Pax6 CKO retinae (Marquardt et al., 2001; OronKarni et al., 2008), indicating that Sufu regulates processes upstream of, and/or parallel to, Pax6 (Oron-Karni et al., 2008). Although progenitors from both Sufu $\mathrm{CKO}$ and Pax6 CKO mice adopt an interneuron fate, the compliment of neuralsubtypes is markedly different. In the Pax6 CKO retina, all neurotransmitter subtypes were made with the exception of GABAergic interneurons (Marquardt et al., 2001). Conversely, dopaminergic neurons, but not glycinergic, cholinergic, or GABAergic neurons were detected in Sufu CKO retinae. A subset of cells within the Sufu-null region is Lim1/2-positive, suggestive of horizontal cell differentiation. However, there is no evidence for horizontal cell differentiation in the Pax6 $\mathrm{CKO}$, although the expression pattern of Lim1 in this model has not been reported. Finally, the consequences of Pax6 and Sufu deletions on RPC proliferation are markedly different. Proliferation is markedly reduced at embryonic stages in Pax6 CKO retina (Marquardt et al., 2001) but not in the Sufu KO retina, which is hyperplastic. This observation also illustrates that regulation of the cell cycle can be separated from the loss of multipotency associated with Pax6 downregulation, which is consistent with previous studies demonstrating the uncoupling of cell fate and proliferation in Hh pathway mutants (Sakagami et al., 2009). Therefore, although the observed downregulation of Pax6 certainly contributes to the overall Sufu-null phenotype, Sufu likely exerts some Pax6independent effects on proliferation.

Sufu regulates the localization of all three Gli transcription factors (Barnfield et al., 2005) and the posttranslational processing of both Gli2 (Chen et al., 2009) and Gli3 (Chen et al., 2009; Jia et al., 2009). Gli2 is the predominant activator of Hh signaling in vivo, including in RPCs (Bai et al., 2002; Wall et al., 2009); however, the functional importance of Sufu-mediated antagonism of Gli2 in vivo has not been reported. The inability of Gli2 ${ }^{-1-}$ explants to induce Gli-dependent transcriptional activation in the context of $\mathrm{Sufu}$ loss-of-function indicated that Gli2 is required to induce $\mathrm{Hh}$ pathway activation in the absence of Sufu. Genetic deletion of Gli2 in Sufu CKO retinae in vivo partially rescued the expression of transcription factors, such as Pax6 and Rax. Moreover, subsets 

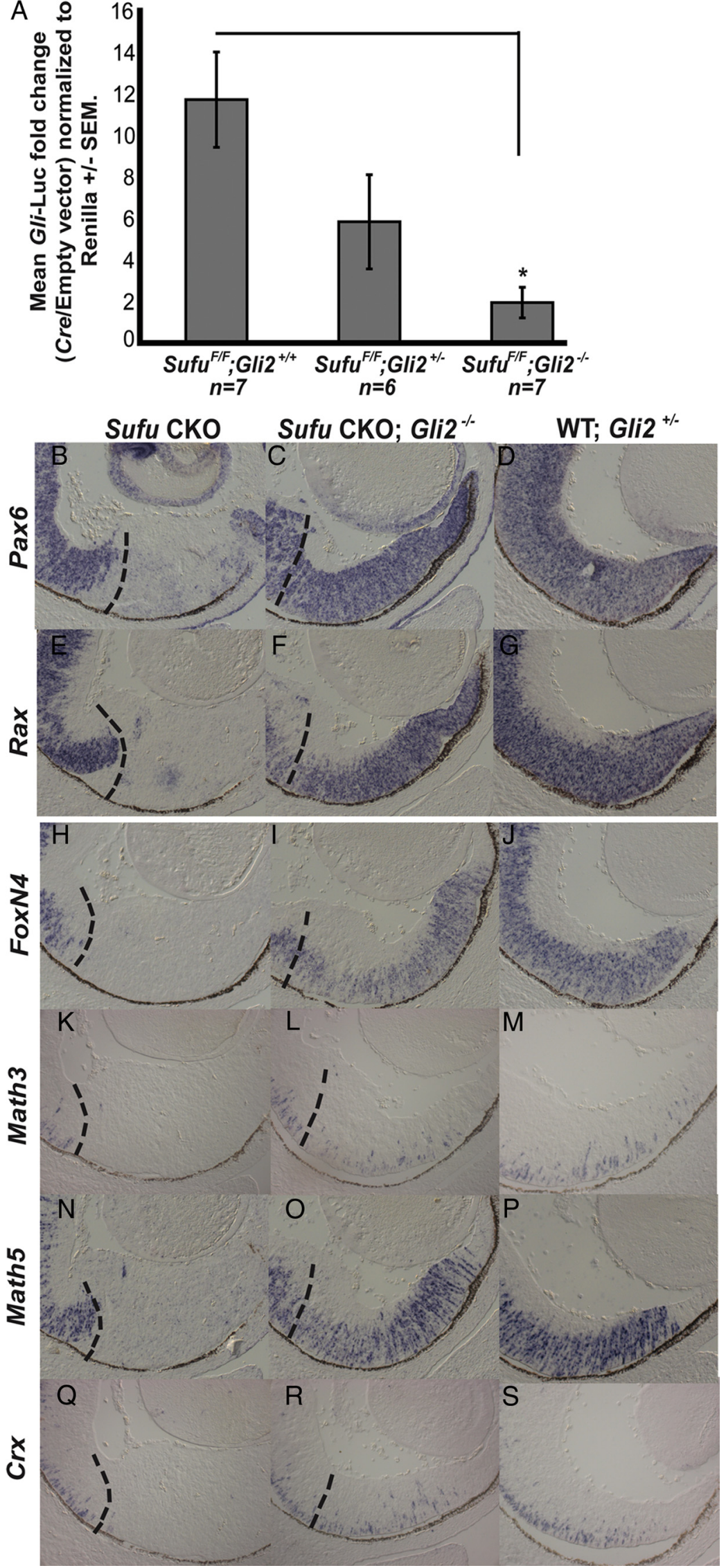

Figure 8. Deletion of Gli2 partially recues the aberrant $H$ h activity and multipotency in Sufu CKO retinae. A, Genetic deletion of Gli2 inhibits Gli-dependent transcription in the context of conditional Sufu deletion. Retinas from PO Sufu ${ }^{F / F} ; G^{\prime i 2}{ }^{+I+}$, Sufu ${ }^{F / F}$; of Sufu CKO;Gli2 ${ }^{-1-}$ RPCs expressed Math5, Math3, and Crx, which are markers of postmitotic retinal precursors (Ohsawa and Kageyama, 2008).Together, these data demonstrate that $S u f u$-mediated antagonism of Gli2 is required to maintain multipotency in early RPCs. Sufu deletion increases the ratio of Gli3 activator:Gli3 repressor (Jia et al., 2009), raising the possibility that deregulated Gli3 activity contributes to the Sufu CKO phenotype in the retina. However, we observed no difference in the phenotypes of Sufu CKO;Gli3 ${ }^{+/-}$retinae compared with Sufu CKO retinae (Cwinn and Wallace, unpublished observations). Additionally, the RPCs of Gli3 ${ }^{-/-}$mice, which also exhibit a $\mathrm{Hh}$ gain-of-function, presumably due to a loss of Gli3-repressor activity (Wang et al., 2000), express Pax6 and $V s \times 2$ (Furimsky and Wallace, 2006), indicating that Gli3 repressor is not required to maintain RPC identity. Although the partial rescue in Sufu $\mathrm{CKO} ; \mathrm{Gli}^{-1-}$ retinae could be due to an increase in Gli3 activator (Jia et al., 2009) or compensation by Gli1 (Kogerman et al., 1999; Taylor et al., 2002; Barnfield et al., 2005), our data strongly suggest that Gli2 is the principal mediator of $\mathrm{Hh}$ signaling in the absence of Sufu in RPCs.

At early stages in retinal development, induction of Hh signaling lags behind the central to peripheral wave of GC differentiation (Wang et al., 2005). Our findings suggest that Sufu is required in RPCs located ahead of the differentiation wave to maintain RPC multipotency. At later stages in retinal development, Sufu function is still required to control $\mathrm{Hh}$ activation and RPC proliferation. In the developing spinal cord, the intensity of Hh signaling in progenitors is determined by their position within the ventral to dorsal gradient of the Shh protein from its source in the ventral floor plate, with the most ventral cells having the highest level of signaling (Jacob and Briscoe, 2003). In this context, Sufu deletion mimics a high Shh signal and causes cells in the neural tube to adopt the most ventral cell

$\leftarrow$

$\mathrm{Gli}^{+I-}$, or Sufu ${ }^{\mathrm{FFF}} ; \mathrm{Gli}^{-/-}$mice were coelectroporated with expression vectors for Gli-luc, Renilla luiferase, Cre or Gli-luc, and Renilla and an empty vector and cultured as explants for $2.5 \mathrm{DIV}$ and $5 \mathrm{DIV}$, and luciferase activity was measured. Error bars represent SEM. ${ }^{*} p<0.05$. $\boldsymbol{B}-\boldsymbol{S}$, Genetic deletion of Gli2 rescues multipotency in Sufu-null RPCs in vivo. In situ hybridization for Pax6 (B-D), Rax (E-G), FoxN4 (H-J), Math3 (K$\mathbf{M})$, Math5 (N-P), and Crx (Q-S) in the retinae of Sufu CKO; $\mathrm{Gli}^{+/+}$, Sufu CKO;Gli2 ${ }^{-/-}$, and $\mathrm{Gli}^{+/-}$mice at E14.5. There was no difference in gene expression between $\mathrm{Gli}^{+/+}$ and Gli2 ${ }^{+/-}$mice (data not shown). Dashed lines delineate the border of the mutant region in the ventral peripheral retina. 
fates (Svard et al., 2006). Because RPCs and Shh-expressing GCs are equidistant in the retina, it is less obvious how graded responses to Shh are achieved in this tissue. Nonetheless, it is tempting to speculate that the consequences of Sufu deletion in embryonic RPC, namely amacrine cell development, mimic the highest level of Shh signaling. In summary, the data presented here implicates Sufu as a novel regulator of the Hh pathway in RPCs and further confirms that Sufu is a critical antagonist of the $H h$ pathway in mammalian systems. The unexpected requirement for Sufu in the maintenance of early RPC multipotency suggests that Sufu-mediated antagonism of Gli 2 is required to mediate the intrinsic behavior of RPCs in response to extrinsic cues.

\section{References}

Alcedo J, Ayzenzon M, Von Ohlen T, Noll M, Hooper JE (1996) The drosophila smoothened gene encodes a seven-pass membrane protein, a putative receptor for the hedgehog signal. Cell 86:221-232.

Amato MA, Boy S, Perron M (2004) Hedgehog signaling in vertebrate eye development: a growing puzzle. Cell Mol Life Sci 61:899-910.

Bai CB, Auerbach W, Lee JS, Stephen D, Joyner AL (2002) Gli2, but not Gli1, is required for initial shh signaling and ectopic activation of the shh pathway. Development 129:4753-4761.

Barnfield PC, Zhang X, Thanabalasingham V, Yoshida M, Hui CC (2005) Negative regulation of Gli1 and Gli2 activator function by suppressor of fused through multiple mechanisms. Differentiation 73:397-405.

Brown NL, Patel S, Brzezinski J, Glaser T (2001) Math5 is required for retinal ganglion cell and optic nerve formation. Development 128:2497-2508.

Burmeister M, Novak J, Liang MY, Basu S, Ploder L, Hawes NL, Vidgen D, Hoover F, Goldman D, Kalnins VI, Roderick TH, Taylor BA, Hankin MH, McInnes RR (1996) Ocular retardation mouse caused by Chx10 homeobox null allele: Impaired retinal progenitor proliferation and bipolar cell differentiation. Nat Genet 12:376-384.

Chen MH, Wilson CW, Li YJ, Law KK, Lu CS, Gacayan R, Zhang X, Hui CC, Chuang PT (2009) Cilium-independent regulation of gli protein function by sufu in hedgehog signaling is evolutionarily conserved. Genes Dev 23:1910-1928.

Cooper AF, Yu KP, Brueckner M, Brailey LL, Johnson L, McGrath JM, Bale AE (2005) Cardiac and CNS defects in a mouse with targeted disruption of suppressor of fused. Development 132:4407-4417.

Demb JB (2007) Cellular mechanisms for direction selectivity in the retina. Neuron 55:179-186.

Ding Q, Fukami S, Meng X, Nishizaki Y, Zhang X, Sasaki H, Dlugosz A, Nakafuku M, Hui C (1999) Mouse suppressor of fused is a negative regulator of sonic hedgehog signaling and alters the subcellular distribution of Gli1. Curr Biol 9:1119-1122.

Dyer MA, Cepko CL (2000) p57(Kip2) regulates progenitor cell proliferation and amacrine interneuron development in the mouse retina. Development 127:3593-3605.

Ericson J, Rashbass P, Schedl A, Brenner-Morton S, Kawakami A, van Heyningen V, Jessell TM, Briscoe J (1997) Pax6 controls progenitor cell identity and neuronal fate in response to graded shh signaling. Cell 90:169-180.

Furimsky M, Wallace VA (2006) Complementary gli activity mediates early patterning of the mouse visual system. Dev Dyn 235:594-605.

Furukawa T, Morrow EM, Li T, Davis FC, Cepko CL (1999) Retinopathy and attenuated circadian entrainment in Crx deficient mice. Nat Genet 23: $466-470$.

Furukawa T, Mukherjee S, Bao ZZ, Morrow EM, Cepko CL (2000) Rax, Hes1, and notch1 promote the formation of muller glia by postnatal retinal progenitor cells. Neuron 26:383-394.

Green ES, Stubbs JL, Levine EM (2003) Genetic rescue of cell number in a mouse model of microphthalmia: Interactions between Chx10 and G1phase cell cycle regulators. Development 130:539-552.

Humke EW, Dorn KV, Milenkovic L, Scott MP, Rohatgi R (2010) The output of hedgehog signaling is controlled by the dynamic association between suppressor of fused and the gli proteins. Genes Dev 24:670-682.

Jacob J, Briscoe J (2003) Gli proteins and the control of spinal-cord patterning. EMBO Rep 4:761-765.

Jensen AM, Wallace VA (1997) Expression of sonic hedgehog and its putative role as a precursor cell mitogen in the developing mouse retina. Development 124:363-371.
Jia J, Kolterud A, Zeng H, Hoover A, Teglund S, Toftgård R, Liu A (2009) Suppressor of fused inhibits mammalian hedgehog signaling in the absence of cilia. Dev Biol 330:452-460.

Kogerman P, Grimm T, Kogerman L, Krause D, Undén AB, Sandstedt B, Toftgård R, Zaphiropoulos PG (1999) Mammalian suppressor-offused modulates nuclear-cytoplasmic shuttling of gli-1. Nat Cell Biol $1: 312-319$.

Lee HY, Wroblewski E, Philips GT, Stair CN, Conley K, Reedy M, Mastick GS, Brown NL (2005) Multiple requirements for hes 1 during early eye formation. Dev Biol 284:464-478.

Lee J, Platt KA, Censullo P, Ruiz i Altaba A (1997) Gli1 is a target of sonic hedgehog that induces ventral neural tube development. Development 124:2537-2552.

Li S, Mo Z, Yang X, Price SM, Shen MM, Xiang M (2004) Foxn4 controls the genesis of amacrine and horizontal cells by retinal progenitors. Neuron 43:795-807.

Li X, Perissi V, Liu F, Rose DW, Rosenfeld MG (2002) Tissue-specific regulation of retinal and pituitary precursor cell proliferation. Science 297:1180-1183

Livne-Bar I, Pacal M, Cheung MC, Hankin M, Trogadis J, Chen D, Dorval KM, Bremner R (2006) Chx10 is required to block photoreceptor differentiation but is dispensable for progenitor proliferation in the postnatal retina. Proc Natl Acad Sci U S A 103:4988-4993.

Locker M, Agathocleous M, Amato MA, Parain K, Harris WA, Perron M (2006) Hedgehog signaling and the retina: Insights into the mechanisms controlling the proliferative properties of neural precursors. Genes Dev 20:3036-3048.

Marquardt T, Ashery-Padan R, Andrejewski N, Scardigli R, Guillemot F, Gruss P (2001) Pax6 is required for the multipotent state of retinal progenitor cells. Cell 105:43-55.

Mathers PH, Grinberg A, Mahon KA, Jamrich M (1997) The rx homeobox gene is essential for vertebrate eye development. Nature 387:603-607.

Matsuda T, Cepko CL (2004) Electroporation and RNA interference in the rodent retina in vivo and in vitro. Proc Natl Acad Sci U S A 101:16-22.

Mo R, Freer AM, Zinyk DL, Crackower MA, Michaud J, Heng HH, Chik KW, Shi XM, Tsui LC, Cheng SH, Joyner AL, Hui C (1997) Specific and redundant functions of Gli2 and Gli3 zinc finger genes in skeletal patterning and development. Development 124:113-123.

Murone M, Rosenthal A, de Sauvage FJ (1999) Sonic hedgehog signaling by the patched-smoothened receptor complex. Curr Biol 9:76-84.

Nguyen-Legros J, Versaux-Botteri C, Savy C (1997) Dopaminergic and GABAergic retinal cell populations in mammals. Microsc Res Tech 36:26-42.

Ohsawa R, Kageyama R (2008) Regulation of retinal cell fate specification by multiple transcription factors. Brain Res 1192:90-98.

Oron-Karni V, Farhy C, Elgart M, Marquardt T, Remizova L, Yaron O, Xie Q, Cvekl A, Ashery-Padan R (2008) Dual requirement for Pax6 in retinal progenitor cells. Development 135:4037-4047.

Pospisilik JA, Schramek D, Schnidar H, Cronin SJ, Nehme NT, Zhang X, Knauf C, Cani PD, Aumayr K, Todoric J, Bayer M, Haschemi A, Puviindran V, Tar K, Orthofer M, Neely GG, Dietzl G, Manoukian A, Funovics M, Prager G, et al. (2010) Drosophila genome-wide obesity screen reveals hedgehog as a determinant of brown versus white adipose cell fate. Cell 140:148-160.

Ruel L, Thérond PP (2009) Variations in hedgehog signaling: Divergence and perpetuation in sufu regulation of gli. Genes Dev 23:1843-1848.

Sakagami K, Gan L, Yang XJ (2009) Distinct effects of hedgehog signaling on neuronal fate specification and cell cycle progression in the embryonic mouse retina. J Neurosci 29:6932-6944.

Sasaki H, Hui C, Nakafuku M, Kondoh H (1997) A binding site for gli proteins is essential for HNF-3beta floor plate enhancer activity in transgenics and can respond to shh in vitro. Development 124:1313-1322.

Shkumatava A, Neumann CJ (2005) Shh directs cell-cycle exit by activating p57Kip2 in the zebrafish retina. EMBO Rep 6:563-569.

Svärd J, Heby-Henricson K, Persson-Lek M, Rozell B, Lauth M, Bergström A, Ericson J, Toftgård R, Teglund S (2006) Genetic elimination of suppressor of fused reveals an essential repressor function in the mammalian hedgehog signaling pathway. Dev Cell 10:187-197.

Taranova OV, Magness ST, Fagan BM, Wu Y, Surzenko N, Hutton SR, Pevny 
LH (2006) SOX2 is a dose-dependent regulator of retinal neural progenitor competence. Genes Dev 20:1187-1202.

Taylor MD, Liu L, Raffel C, Hui CC, Mainprize TG, Zhang X, Agatep R, Chiappa S, Gao L, Lowrance A, Hao A, Goldstein AM, Stavrou T, Scherer SW, Dura WT, Wainwright B, Squire JA, Rutka JT, Hogg D (2002) Mutations in SUFU predispose to medulloblastoma. Nat Genet 31:306-310.

Varjosalo M, Li SP, Taipale J (2006) Divergence of hedgehog signal transduction mechanism between drosophila and mammals. Dev Cell 10:177-186.

Wall DS, Mears AJ, McNeill B, Mazerolle C, Thurig S, Wang Y, Kageyama R, Wallace VA (2009) Progenitor cell proliferation in the retina is dependent on notch-independent sonic hedgehog/Hesl activity. J Cell Biol 184:101-112.

Wang B, Fallon JF, Beachy PA (2000) Hedgehog-regulated processing of Gli3 produces an anterior/posterior repressor gradient in the developing vertebrate limb. Cell 100:423-434.

Wang C, Pan Y, Wang B (2010) Suppressor of fused and spop regulate the stability, processing and function of Gli2 and Gli3 full-length activators but not their repressors. Development 137:2001-2009.

Wang SW, Kim BS, Ding K, Wang H, Sun D, Johnson RL, Klein WH, Gan L
(2001) Requirement for math5 in the development of retinal ganglion cells. Genes Dev 15:24-29.

Wang Y, Dakubo GD, Thurig S, Mazerolle CJ, Wallace VA (2005) Retinal ganglion cell-derived sonic hedgehog locally controls proliferation and the timing of RGC development in the embryonic mouse retina. Development 132:5103-5113.

Xie J, Murone M, Luoh SM, Ryan A, Gu Q, Zhang C, Bonifas JM, Lam CW, Hynes M, Goddard A, Rosenthal A, Epstein EH Jr, de Sauvage FJ (1998) Activating smoothened mutations in sporadic basal-cell carcinoma. Nature 391:90-92.

Xu S, Sunderland ME, Coles BL, Kam A, Holowacz T, Ashery-Padan R, Marquardt T, McInnes RR, van der Kooy D (2007) The proliferation and expansion of retinal stem cells require functional Pax6. Dev Biol 304:713-721.

Young RW (1985) Cell differentiation in the retina of the mouse. Anat Rec 212:199-205.

Yu C, Mazerolle CJ, Thurig S, Wang Y, Pacal M, Bremner R, Wallace VA (2006) Direct and indirect effects of hedgehog pathway activation in the mammalian retina. Mol Cell Neurosci 32:274-282.

Zhang L, Mathers PH, Jamrich M (2000) Function of rx, but not Pax6, is essential for the formation of retinal progenitor cells in mice. Genesis $28: 135-142$. 